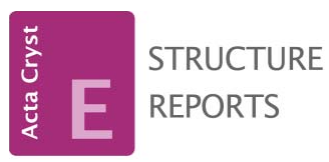

ISSN 1600-5368

Received 13 November 2014 Accepted 26 November 2014

Edited by M. Weil, Vienna University of Technology, Austria

Keywords: crystal structure; $\mathrm{TiNiSi}$ structure type; six-membered rings of strontium; intermetallic compound

CCDC reference: 1036051

Supporting information: this article has supporting information at journals.iucr.org/e

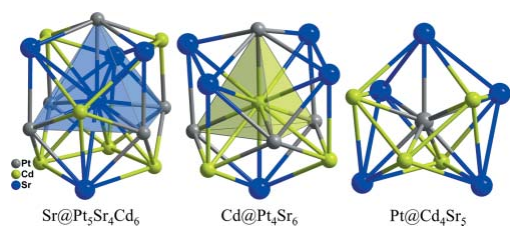

OPEN $\odot$ ACCESS

\section{Crystal structure of the intermetallic compound SrCdPt}

\author{
Fakhili Gulo $^{\mathrm{a} *}$ and Jürgen Köhler ${ }^{\mathrm{b}}$ \\ aDepartment of Chemical Education, Sriwijaya University, Inderalaya, Ogan Ilir 30662, South Sumatra, Indonesia, and \\ b Max Planck Institut für Festkörperforschung, Heisenbergstrasse 1, 70698 Stuttgart, Germany. *Correspondence e-mail: \\ fgulo@unsri.ac.id
}

The crystal structure of the title compound, strontium cadmium platinum, adopts the TiNiSi structure type with the Sr atoms on the Ti, the Cd atoms on the $\mathrm{Ni}$ and the $\mathrm{Pt}$ atoms on the $\mathrm{Si}$ positions, respectively. The $\mathrm{Pt}$ atoms form cadmium-centred tetrahedra that are condensed into a three-dimensional network with channels parallel to the $b$-axis direction in which the $\mathrm{Sr}$ atoms are located. The latter are bonded to each other in the form of six-membered rings with chair conformations. All atoms in the SrCdPt structure are situated on a mirror plane.

\section{Chemical context}

Exploratory synthesis of polar intermetallic phases has proven to be productive in terms of novel compositions, new and unprecedented structures, and unusual bonding regimes (Corbett, 2010). Platinum has participated significantly in the formation of ternary intermetallic compounds. Together with indium, a number of platinum phases have been reported, for example BaPtIn 3 (Palasyuk \& Corbett, 2007), SrPtIn (Hoffmann \& Pöttgen, 1999), CaPtIn 2 (Hoffmann et al., 1999) or $\mathrm{Ca}_{2} \mathrm{Pt}_{2}$ In (Muts et al., 2007). Some other ternary intermetallic compounds of platinum with cadmium, viz. $\mathrm{Ca}_{2} \mathrm{CdPt}_{2}$ (Samal \& Corbett, 2012), $\mathrm{Ca}_{6} \mathrm{Pt}_{8} \mathrm{Cd}_{16},(\mathrm{Ba} / \mathrm{Sr}) \mathrm{Cd}_{4} \mathrm{Pt}_{2}$ (Samal et al., 2013), $\mathrm{Ca}_{6} \mathrm{Cd}_{11} \mathrm{Pt}$ (Gulo et al., 2013) and CaCdPt (Kersting et $a l ., 2013$ ) have been isolated recently. They demonstrate the diversity of the structures types adopted. In this communication, we present the crystal structure of SrCdPt.

\section{Structural commentary}

SrCdPt crystallizes in the TiNiSi structure type. The titanium, nickel, and silicon sites are occupied by strontium, cadmium, and platinum, respectively, in the structure of the title compound. Although platinum and nickel are in the same group in the periodic table, the platinum in SrCdPt occupies the silicon site and not the nickel site because platinum is the most electronegative metal in this structure, just like silicon in TiNiSi. A count of 56 valence electrons per cell is found in SrCdPt $[(\mathrm{Sr}: 2+\mathrm{Cd}: 2+\mathrm{Pt}: 10) \times 4]$ whilst TiNiSi contains only 32 valence electrons per cell.

In the compounds of the TiNiSi structure family, the metals listed first in the formula are linked to each other, forming sixmembered rings in chair, half-chair, or boat conformations. The adopted conformation is not a function of the electron count, but is due to the nature of the respective metal (Landrum et al., 1998). In the SrCdPt structure, the strontium atoms construct six-membered rings with chair conformations 


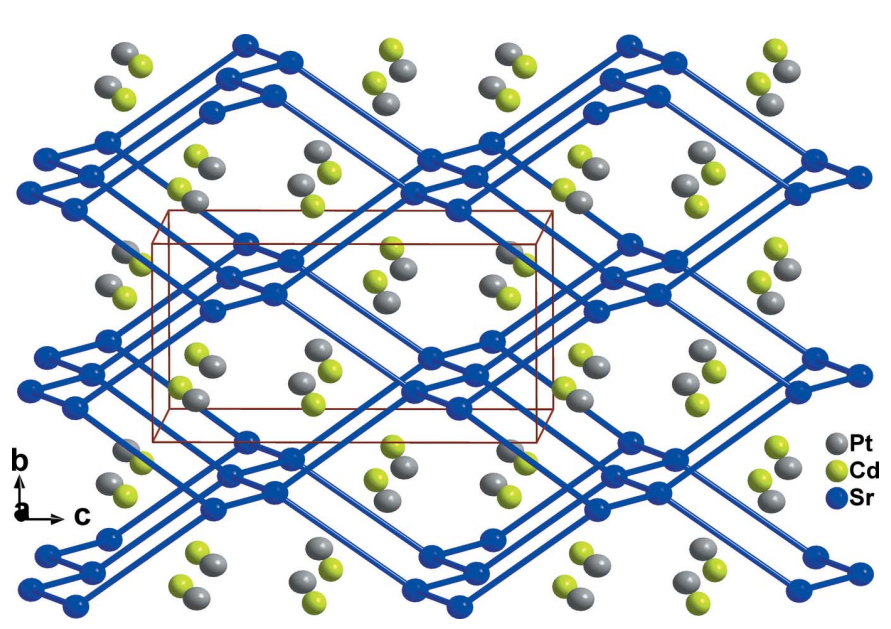

Figure 1

Projection of the crystal structure of SrCdPt approximately along [100]. Displacement ellipsoids are represented at the $90 \%$ probability level.

and $\mathrm{Sr}-\mathrm{Sr}$ distances of 3.870 (2) $\AA$, which is significantly shorter than the sum of the covalent radii of $4.30 \AA$ (Emsley, 1999), indicating strong bonding interactions between them (Fig. 1). The existence of such strong $\mathrm{Sr}-\mathrm{Sr}$ bonds is not noticeable in $\mathrm{SrCd}_{4} \mathrm{Pt}_{2}$ (Samal et al., 2013). The platinum atoms in the structure of SrCdPt form zigzag chains of edgesharing cadmium-centred tetrahedra parallel to the $b$-axis direction. These chains are condensed via common corners with adjacent chains, building up the three-dimensional network with channels parallel to the $b$-axis direction in which the $\mathrm{Sr}$ atoms reside, as illustrated in Fig. 2.

Strontium has an overall coordination number of 15 and is surrounded by four other strontium, six cadmium, and five

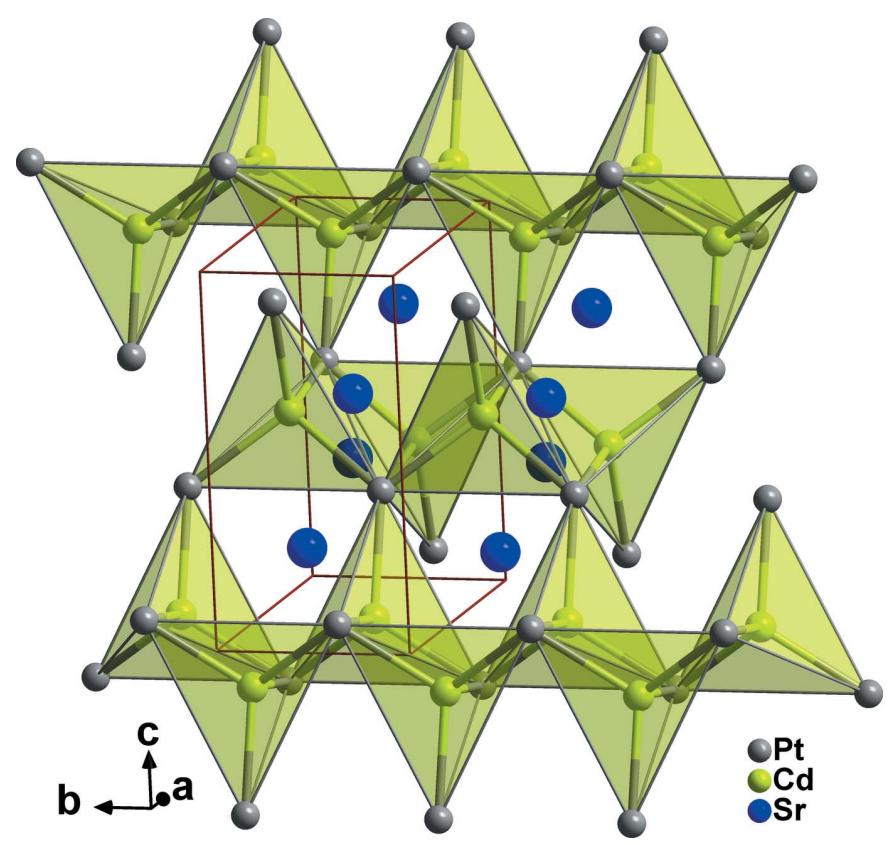

Figure 2

View of zigzag chains of cadmium-centred tetrahedra of Pt atoms forming channels along the $b$-axis direction in the structure of SrCdPt.
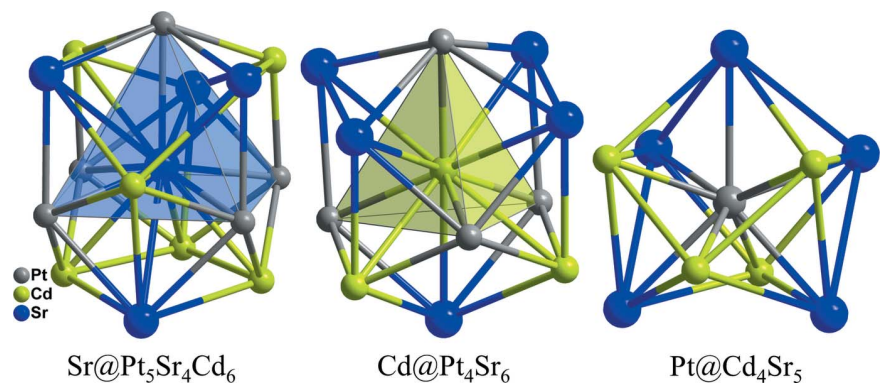

Figure 3

Coordination polyhedra of $\mathrm{Sr}, \mathrm{Cd}$, and $\mathrm{Pt}$ atoms in the structure of SrCdPt.

platinum atoms. The $\mathrm{Sr}-\mathrm{Cd}$ distances range from 3.3932 (13) to 3.6124 (17) $\AA$, whereas the $\mathrm{Sr}-\mathrm{Pt}$ distances vary only slightly, from 3.1943 (11) to 3.2238 (10) $\AA$. Cadmium is located at a site that is surrounded by six strontium and four platinum atoms, whilst platinum has a coordination number of 9 defined by five strontium and four cadmium atoms. The environment of each atom in this structure is represented in Fig. 3. The interatomic distances $(\mathrm{Sr}-\mathrm{Cd}, \mathrm{Sr}-\mathrm{Pt}$, and $\mathrm{Cd}-\mathrm{Pt})$ are in good agreement with those found in the structures of some other ternary compounds in the alkaline earth-Cd-Pt system (Samal \& Corbett, 2012; Samal et al., 2013; Gulo et al., 2013; Kersting et al., 2013). In SrCdPt, the shortest $\mathrm{Cd}-\mathrm{Cd}$ distance of 3.3197 (15) $\AA$ is too long to be considered as a bond. It is significantly longer than the sum of the covalent radii of $2.90 \AA$ (Emsley, 1999). In contrast, cadmium atoms are bonded together, forming $\mathrm{Cd}_{4}$ tetrahedra in $\mathrm{SrCd}_{4} \mathrm{Pt}_{2}, \mathrm{Cd}_{8}$ tetrahedral stars in $\mathrm{Ca}_{6} \mathrm{Cd}_{16} \mathrm{Pt}_{8}$, and $\mathrm{Cd}_{7}$ pentagonal bipyramids in $\mathrm{Ca}_{6} \mathrm{Cd}_{11} \mathrm{Pt}$.

\section{Database survey}

A search of the Pearson's Crystal Data - Crystal Structure Database for Inorganic Compounds (Villars \& Cenzual, 2011) for the TiNiSi family of compounds returned 1101 entries with the same prototype. Two ternary compounds of them include strontium and platinum, one compound includes strontium with cadmium, and no compound had formed so far including both cadmium and platinum.

\section{Synthesis and crystallization}

Starting materials for the synthesis of the title compound were ingots of strontium (99.9+\%, Alfa Aesar), cadmium powder (99.9+\%, Alfa Aesar) and platinum powder (99.95\%, Chempur). A stoichiometric mixture of these elements was weighed and loaded into a tantalum ampoule in an argon-filled dry box. The tantalum ampoule was then weld-sealed under an argon atmosphere and subsequently enclosed in an evacuated silica jacket. The sample was then heated to $1123 \mathrm{~K}$ for $15 \mathrm{~h}$, followed by equilibration at $923 \mathrm{~K}$ for 4 days, and slow cooling to room temperature. The synthesis procedures were similar to general methods applied in some previous experiments (Gulo et al., 2013). 
Table 1

Experimental details.

\begin{tabular}{|c|c|}
\hline \multicolumn{2}{|l|}{ Crystal data } \\
\hline Chemical formula & $\mathrm{SrCdPt}$ \\
\hline$M_{\mathrm{r}}$ & 395.11 \\
\hline Crystal system, space group & Orthorhombic, Pnma \\
\hline Temperature (K) & 298 \\
\hline$a, b, c(\AA)$ & $7.5748(15), 4.4774(9), 8.6383$ (17) \\
\hline$V\left(\AA^{3}\right)$ & $292.97(10)$ \\
\hline$Z$ & 4 \\
\hline Radiation type & Мo $K \alpha$ \\
\hline$\mu\left(\mathrm{mm}^{-1}\right)$ & 72.61 \\
\hline Crystal size (mm) & $0.05 \times 0.04 \times 0.03$ \\
\hline \multicolumn{2}{|l|}{ Data collection } \\
\hline Diffractometer & Bruker $S M A R T$ CCD \\
\hline Absorption correction & $\begin{array}{l}\text { Multi-scan ( } S A D A B S \text {; Bruker, } \\
\text { 2001) }\end{array}$ \\
\hline$T_{\min }, T_{\max }$ & $0.043,0.113$ \\
\hline $\begin{array}{l}\text { No. of measured, independent and } \\
\text { observed }[I>2 \sigma(I)] \text { reflections }\end{array}$ & $2231,381,338$ \\
\hline$R_{\text {int }}$ & 0.061 \\
\hline$(\sin \theta / \lambda)_{\max }\left(\AA^{-1}\right)$ & 0.664 \\
\hline \multicolumn{2}{|l|}{ Refinement } \\
\hline$R\left[F^{2}>2 \sigma\left(F^{2}\right)\right], w R\left(F^{2}\right), S$ & $0.030,0.066,1.07$ \\
\hline No. of reflections & 381 \\
\hline No. of parameters & 19 \\
\hline$\Delta \rho_{\max }, \Delta \rho_{\min }\left(\mathrm{e} \AA^{-3}\right)$ & $2.22,-1.87$ \\
\hline
\end{tabular}

Computer programs: SMART and SAINT (Bruker, 2001), SHELXS97 and SHELXL97 (Sheldrick, 2008) and DIAMOND (Brandenburg, 2006).

\section{Refinement}

Crystal data, data collection and structure refinement details are summarized in Table 1 . The highest remaining electron density is located $0.98 \AA$ from the Pt site.

\section{Acknowledgements}

Financial support for FG from PNBP Unsri is gratefully acknowledged.

\section{References}

Brandenburg, K. (2006). DIAMOND. Crystal Impact GbR, Bonn, Germany.

Bruker (2001). SMART, SAINT and SADABS. Bruker AXS Inc., Madison, Wisconsin, USA.

Corbett, J. D. (2010). Inorg. Chem. 49, 13-28.

Emsley, J. (1999). In The Elements. Oxford University Press.

Gulo, F., Samal, S. L. \& Corbett, J. D. (2013). Inorg. Chem. 52, 1011210118.

Hoffmann, R. D. \& Pöttgen, R. (1999). Z. Anorg. Allg. Chem. 625, 994-1000.

Hoffmann, R. D., Pöttgen, R., Landrum, G. A., Dronskowski, R., Künnen, B. \& Kotzyba, G. (1999). Z. Anorg. Allg. Chem. 625, 789798.

Kersting, M., Johnscher, M. \& Pöttgen, R. (2013). Z. Kristallogr. 228, 635-642.

Landrum, G. A., Hoffmann, R., Evers, J. \& Boysen, H. (1998). Inorg. Chem. 37, 5754-5763.

Muts, I. R., Zaremba, V. I., Rodewald, U. C., Hermes, W. \& Pöttgen, R. (2007). Z. Anorg. Allg. Chem. 633, 2725-2729.

Palasyuk, A. \& Corbett, J. D. (2007). Z. Anorg. Allg. Chem. 633, 2563-2567.

Samal, S. L. \& Corbett, J. D. (2012). Z. Anorg. Allg. Chem. 638, $1963-$ 1969.

Samal, S. L., Gulo, F. \& Corbett, J. D. (2013). Inorg. Chem. 52, $2697-$ 2704.

Sheldrick, G. M. (2008). Acta Cryst. A64, 112-122.

Villars, P. \& Cenzual, K. (2011). Pearson's Crystal Data-Crystal Structure Database for Inorganic Compounds, Release 2010/11. ASM International, Materials Park, Ohio, USA. 


\section{supporting information}

Acta Cryst. (2014). E70, 590-592 [doi:10.1107/S1600536814025823]

\section{Crystal structure of the intermetallic compound SrCdPt}

\section{Fakhili Gulo and Jürgen Köhler}

\section{Computing details}

Data collection: SMART (Bruker, 2001); cell refinement: SAINT (Bruker, 2001); data reduction: SAINT (Bruker, 2001); program(s) used to solve structure: SHELXS97 (Sheldrick, 2008); program(s) used to refine structure: SHELXL97 (Sheldrick, 2008); molecular graphics: DIAMOND (Brandenburg, 2006); software used to prepare material for publication: SHELXL97 (Sheldrick, 2008).

\section{Strontium cadmium platinum}

Crystal data

$\mathrm{SrCdPt}$

$M_{r}=395.11$

Orthorhombic, Pnma

Hall symbol: -P 2ac 2n

$a=7.5748(15) \AA$

$b=4.4774(9) \AA$

$c=8.6383(17) \AA$

$V=292.97(10) \AA^{3}$

$Z=4$

Data collection

Bruker SMART CCD

diffractometer

Radiation source: fine-focus sealed tube

Graphite monochromator

Detector resolution: 0 pixels $\mathrm{mm}^{-1}$

$\omega$ scans

Absorption correction: multi-scan

(SADABS; Bruker, 2001)

$T_{\min }=0.043, T_{\max }=0.113$

Refinement

Refinement on $F^{2}$

Least-squares matrix: full

$R\left[F^{2}>2 \sigma\left(F^{2}\right)\right]=0.030$

$w R\left(F^{2}\right)=0.066$

$S=1.07$

381 reflections

19 parameters

0 restraints
$F(000)=656$

$D_{\mathrm{x}}=8.958 \mathrm{Mg} \mathrm{m}^{-3}$

Mo $K \alpha$ radiation, $\lambda=0.71073 \AA$

Cell parameters from 25 reflections

$\theta=12-18^{\circ}$

$\mu=72.61 \mathrm{~mm}^{-1}$

$T=298 \mathrm{~K}$

Block, brown

$0.05 \times 0.04 \times 0.03 \mathrm{~mm}$

2231 measured reflections

381 independent reflections

338 reflections with $I>2 \sigma(I)$

$R_{\text {int }}=0.061$

$\theta_{\max }=28.1^{\circ}, \theta_{\min }=3.6^{\circ}$

$h=-9 \rightarrow 9$

$k=-5 \rightarrow 5$

$l=-11 \rightarrow 11$

Primary atom site location: structure-invariant direct methods

Secondary atom site location: difference Fourier map

$w=1 /\left[\sigma^{2}\left(F_{\mathrm{o}}^{2}\right)+(0.0307 P)^{2}\right]$

where $P=\left(F_{\mathrm{o}}{ }^{2}+2 F_{\mathrm{c}}{ }^{2}\right) / 3$

$(\Delta / \sigma)_{\max }<0.001$

$\Delta \rho_{\max }=2.22 \mathrm{e} \AA^{-3}$

$\Delta \rho_{\min }=-1.87$ e $\AA^{-3}$ 


\section{Special details}

Geometry. All e.s.d.'s (except the e.s.d. in the dihedral angle between two 1.s. planes) are estimated using the full covariance matrix. The cell e.s.d.'s are taken into account individually in the estimation of e.s.d.'s in distances, angles and torsion angles; correlations between e.s.d.'s in cell parameters are only used when they are defined by crystal symmetry. An approximate (isotropic) treatment of cell e.s.d.'s is used for estimating e.s.d.'s involving 1.s. planes.

Refinement. Refinement of $F^{2}$ against ALL reflections. The weighted $R$-factor $w R$ and goodness of fit $S$ are based on $F^{2}$, conventional $R$-factors $R$ are based on $F$, with $F$ set to zero for negative $F^{2}$. The threshold expression of $F^{2}>\sigma\left(F^{2}\right)$ is used only for calculating $R$-factors (gt) etc. and is not relevant to the choice of reflections for refinement. $R$-factors based on $F^{2}$ are statistically about twice as large as those based on $F$, and $R$ - factors based on ALL data will be even larger.

Fractional atomic coordinates and isotropic or equivalent isotropic displacement parameters $\left(\hat{A}^{2}\right)$

\begin{tabular}{lllll}
\hline & $x$ & $y$ & $z$ & $U_{\text {iso }} * / U_{\text {eq }}$ \\
\hline $\mathrm{Pt}$ & $0.27016(7)$ & 0.2500 & $0.37717(7)$ & $0.0150(2)$ \\
$\mathrm{Cd}$ & $0.14353(12)$ & 0.2500 & $0.06550(12)$ & $0.0140(3)$ \\
$\mathrm{Sr}$ & $0.02883(16)$ & 0.2500 & $0.68094(16)$ & $0.0141(3)$ \\
\hline
\end{tabular}

Atomic displacement parameters $\left(\AA^{2}\right)$

\begin{tabular}{lllllll}
\hline & $U^{11}$ & $U^{22}$ & $U^{33}$ & $U^{12}$ & $U^{13}$ & $U^{23}$ \\
\hline $\mathrm{Pt}$ & $0.0175(3)$ & $0.0114(3)$ & $0.0160(4)$ & 0.000 & $0.0005(2)$ & 0.000 \\
$\mathrm{Cd}$ & $0.0173(6)$ & $0.0120(5)$ & $0.0127(6)$ & 0.000 & $0.0013(4)$ & 0.000 \\
$\mathrm{Sr}$ & $0.0161(7)$ & $0.0116(6)$ & $0.0147(7)$ & 0.000 & $0.0006(5)$ & 0.000 \\
\hline
\end{tabular}

Geometric parameters $\left(\stackrel{A}{\circ}{ }^{\circ}\right)$

\begin{tabular}{|c|c|c|c|}
\hline $\mathrm{Pt}-\mathrm{Cd}^{\mathrm{i}}$ & $2.8435(8)$ & $\mathrm{Cd}-\mathrm{Sr}^{\mathrm{xi}}$ & $3.4336(17)$ \\
\hline $\mathrm{Pt}-\mathrm{Cd}^{\mathrm{ii}}$ & $2.8435(8)$ & $\mathrm{Cd}-\mathrm{Sr}^{\mathrm{v}}$ & 3.4879 (13) \\
\hline $\mathrm{Pt}-\mathrm{Cd}$ & $2.8581(13)$ & $\mathrm{Cd}-\mathrm{Sr}^{\mathrm{iv}}$ & 3.4879 (13) \\
\hline $\mathrm{Pt}-\mathrm{Cd}^{\mathrm{iii}}$ & $2.8713(12)$ & $\mathrm{Cd}-\mathrm{Sr}^{\mathrm{iii}}$ & $3.6124(17)$ \\
\hline $\mathrm{Pt}-\mathrm{Sr}^{\mathrm{iv}}$ & $3.1943(11)$ & $\mathrm{Sr}-\mathrm{Pt}^{\mathrm{i}}$ & $3.1943(11)$ \\
\hline $\mathrm{Pt}-\mathrm{Sr}^{v}$ & $3.1943(11)$ & $\mathrm{Sr}-\mathrm{Pt}^{\mathrm{ii}}$ & $3.1943(11)$ \\
\hline $\mathrm{Pt}-\mathrm{Sr}$ & $3.1980(15)$ & $\mathrm{Sr}-\mathrm{Pt}^{\mathrm{vi}}$ & $3.2238(10)$ \\
\hline $\mathrm{Pt}-\mathrm{Sr}^{\mathrm{vi}}$ & $3.2238(10)$ & $\mathrm{Sr}-\mathrm{Pt}^{\mathrm{vii}}$ & $3.2238(10)$ \\
\hline $\mathrm{Pt}-\mathrm{Sr}^{\mathrm{vii}}$ & $3.2238(10)$ & $\mathrm{Sr}-\mathrm{Cd}^{\mathrm{vii}}$ & $3.3932(13)$ \\
\hline $\mathrm{Cd}-\mathrm{Pt}^{\mathrm{tiv}}$ & $2.8435(8)$ & $\mathrm{Sr}-\mathrm{Cd}^{\mathrm{vi}}$ & $3.3932(13)$ \\
\hline $\mathrm{Cd}-\mathrm{Pt}^{\mathrm{v}}$ & $2.8435(8)$ & $\mathrm{Sr}-\mathrm{Cd}^{\mathrm{xii}}$ & $3.4336(17)$ \\
\hline $\mathrm{Cd}-\mathrm{Pt}^{\mathrm{viii}}$ & $2.8713(12)$ & $\mathrm{Sr}-\mathrm{Cd}^{\mathrm{i}}$ & 3.4879 (13) \\
\hline $\mathrm{Cd}-\mathrm{Cd}^{\mathrm{ix}}$ & $3.3197(15)$ & $\mathrm{Sr}-\mathrm{Cd}^{\mathrm{ii}}$ & $3.4879(13)$ \\
\hline $\mathrm{Cd}-\mathrm{Cd}^{\mathrm{x}}$ & $3.3197(15)$ & $\mathrm{Sr}-\mathrm{Cd}^{\text {viii }}$ & $3.6124(17)$ \\
\hline $\mathrm{Cd}-\mathrm{Sr}^{\mathrm{vii}}$ & $3.3932(13)$ & $\mathrm{Sr}-\mathrm{Sr}^{\mathrm{vi}}$ & $3.870(2)$ \\
\hline $\mathrm{Cd}-\mathrm{Sr}^{\mathrm{vi}}$ & $3.3932(13)$ & & \\
\hline $\mathrm{Cd}-\mathrm{Pt}-\mathrm{Cd}^{\mathrm{ii}}$ & $103.87(4)$ & $\mathrm{Pt}^{\mathrm{viii}}-\mathrm{Cd}-\mathrm{Sr}^{\mathrm{iv}}$ & $130.66(3)$ \\
\hline $\mathrm{Cd}^{\mathrm{i}}-\mathrm{Pt}-\mathrm{Cd}$ & $128.03(2)$ & $\mathrm{Cd}^{\mathrm{ix}}-\mathrm{Cd}-\mathrm{Sr}^{\mathrm{iv}}$ & $97.51(2)$ \\
\hline $\mathrm{Cd}^{\mathrm{ii}}-\mathrm{Pt}-\mathrm{Cd}$ & $128.03(2)$ & $\mathrm{Cd}^{\mathrm{x}}-\mathrm{Cd}-\mathrm{Sr}^{\mathrm{iv}}$ & $175.11(5)$ \\
\hline $\mathrm{Cd}-\mathrm{Pt}-\mathrm{Cd}^{\mathrm{iii}}$ & $71.03(3)$ & $\mathrm{Sr}^{\mathrm{vii}}-\mathrm{Cd}-\mathrm{Sr}^{\mathrm{iv}}$ & $120.84(3)$ \\
\hline $\mathrm{Cd}^{\mathrm{di}}-\mathrm{Pt}-\mathrm{Cd}^{\mathrm{iii}}$ & $71.03(3)$ & $\mathrm{Sr}^{\mathrm{vi}}-\mathrm{Cd}-\mathrm{Sr}^{\mathrm{iv}}$ & $70.47(2)$ \\
\hline $\mathrm{Cd}-\mathrm{Pt}-\mathrm{Cd}^{\mathrm{iii}}$ & $119.54(3)$ & $\mathrm{Sr}^{\mathrm{xi}}-\mathrm{Cd}-\mathrm{Sr}^{\mathrm{iv}}$ & $117.17(3)$ \\
\hline
\end{tabular}




\begin{tabular}{|c|c|c|c|}
\hline $\mathrm{Cd}^{\mathrm{i}}-\mathrm{Pt}-\mathrm{Sr}^{\mathrm{iv}}$ & $138.23(3)$ & $\mathrm{Sr}^{\mathrm{v}}-\mathrm{Cd}-\mathrm{Sr}^{\mathrm{iv}}$ & $79.86(4)$ \\
\hline $\mathrm{Cd}{ }^{\mathrm{ii}}-\mathrm{Pt}-\mathrm{Sr}^{\mathrm{iv}}$ & $69.04(3)$ & $\mathrm{Pt}^{\mathrm{iv}}-\mathrm{Cd}-\mathrm{Sr}^{\mathrm{iii}}$ & $58.47(2)$ \\
\hline $\mathrm{Cd}-\mathrm{Pt}-\mathrm{Sr}^{\mathrm{iv}}$ & $70.13(3)$ & $\mathrm{Pt}^{\mathrm{v}}-\mathrm{Cd}-\mathrm{Sr}^{\mathrm{iii}}$ & $58.47(2)$ \\
\hline $\mathrm{Cd}^{\mathrm{iii}}-\mathrm{Pt}-\mathrm{Sr}^{\mathrm{iv}}$ & $67.79(3)$ & $\mathrm{Pt}-\mathrm{Cd}-\mathrm{Sr}^{\mathrm{iii}}$ & $106.50(4)$ \\
\hline $\mathrm{Cd}-\mathrm{Pt}-\mathrm{Sr}^{\mathrm{v}}$ & $69.04(3)$ & $\mathrm{P}^{\mathrm{viii}}-\mathrm{Cd}-\mathrm{Sr}^{\mathrm{iii}}$ & $153.82(4)$ \\
\hline $\mathrm{Cd}^{\mathrm{ii}}-\mathrm{Pt}-\mathrm{Sr}^{\mathrm{v}}$ & $138.23(3)$ & $\mathrm{Cd}^{\mathrm{ix}}-\mathrm{Cd}-\mathrm{Sr}^{\mathrm{iii}}$ & $109.17(4)$ \\
\hline $\mathrm{Cd}-\mathrm{Pt}-\mathrm{Sr}^{v}$ & $70.13(3)$ & $\mathrm{Cd}^{\mathrm{x}}-\mathrm{Cd}-\mathrm{Sr}^{\mathrm{iii}}$ & $109.17(4)$ \\
\hline $\mathrm{Cd}{ }^{\mathrm{iii}}-\mathrm{Pt}-\mathrm{Sr}^{\mathrm{v}}$ & $67.79(3)$ & $\mathrm{Sr}^{\mathrm{vii}}-\mathrm{Cd}-\mathrm{Sr}^{\mathrm{iii}}$ & $133.73(2)$ \\
\hline $\mathrm{Sr}^{\mathrm{iv}}-\mathrm{Pt}-\mathrm{Sr}^{\mathrm{v}}$ & 88.99 (4) & $\mathrm{Sr}^{\mathrm{vi}}-\mathrm{Cd}-\mathrm{Sr}^{\mathrm{iii}}$ & $133.73(2)$ \\
\hline $\mathrm{Cd}^{\mathrm{i}}-\mathrm{Pt}-\mathrm{Sr}$ & $70.24(3)$ & $\mathrm{Sr}^{\mathrm{xi}}-\mathrm{Cd}-\mathrm{Sr}^{\mathrm{iii}}$ & $68.55(3)$ \\
\hline $\mathrm{Cd}^{\mathrm{di}}-\mathrm{Pt}-\mathrm{Sr}$ & $70.24(3)$ & $\mathrm{Sr}^{\mathrm{v}}-\mathrm{Cd}-\mathrm{Sr}^{\mathrm{iii}}$ & $66.02(3)$ \\
\hline $\mathrm{Cd}-\mathrm{Pt}-\mathrm{Sr}$ & $125.53(4)$ & $\mathrm{Sr}^{\mathrm{iv}}-\mathrm{Cd}-\mathrm{Sr}^{\mathrm{iii}}$ & $66.02(3)$ \\
\hline $\mathrm{Cd}^{\mathrm{iii}}-\mathrm{Pt}-\mathrm{Sr}$ & $114.93(4)$ & $\mathrm{Pt}^{\mathrm{i}}-\mathrm{Sr}-\mathrm{Pt}^{\mathrm{ii}}$ & 88.99 (4) \\
\hline $\mathrm{Sr}^{\mathrm{iv}}-\mathrm{Pt}-\mathrm{Sr}$ & $135.06(2)$ & $\mathrm{Pt}^{\mathrm{i}}-\mathrm{Sr}-\mathrm{Pt}$ & $99.38(3)$ \\
\hline $\mathrm{Sr}^{\mathrm{v}}-\mathrm{Pt}-\mathrm{Sr}$ & $135.06(2)$ & $\mathrm{Pt}^{\mathrm{ti}}-\mathrm{Sr}-\mathrm{Pt}$ & $99.38(3)$ \\
\hline $\mathrm{Cd}^{\mathrm{i}}-\mathrm{Pt}-\mathrm{Sr}^{\mathrm{vi}}$ & $142.88(3)$ & $\mathrm{Pt}^{\mathrm{i}}-\mathrm{Sr}-\mathrm{Pt}^{\mathrm{vi}}$ & $154.71(5)$ \\
\hline $\mathrm{Cd}^{\mathrm{ii}}-\mathrm{Pt}-\mathrm{Sr}^{\mathrm{vi}}$ & $72.78(3)$ & $\mathrm{Pt}^{\mathrm{ii}}-\mathrm{Sr}-\mathrm{Pt}^{\mathrm{vi}}$ & $86.033(18)$ \\
\hline $\mathrm{Cd}-\mathrm{Pt}-\mathrm{Sr}^{\mathrm{vi}}$ & $67.51(3)$ & $\mathrm{Pt}-\mathrm{Sr}-\mathrm{Pt}^{\mathrm{vi}}$ & $105.89(3)$ \\
\hline $\mathrm{Cd}^{\mathrm{iii}}-\mathrm{Pt}-\mathrm{Sr}^{\mathrm{vi}}$ & $135.959(18)$ & $\mathrm{Pt}^{\mathrm{i}}-\mathrm{Sr}-\mathrm{Pt}^{\mathrm{vii}}$ & $86.033(18)$ \\
\hline $\mathrm{Sr}^{\mathrm{iv}}-\mathrm{Pt}-\mathrm{Sr}^{\mathrm{vi}}$ & 76.441 (19) & $\mathrm{Pt}^{\mathrm{ti}}-\mathrm{Sr}-\mathrm{Pt}^{\mathrm{vii}}$ & $154.71(5)$ \\
\hline $\mathrm{Sr}^{\mathrm{v}}-\mathrm{Pt}-\mathrm{Sr}^{\mathrm{vi}}$ & $137.64(2)$ & $\mathrm{Pt}-\mathrm{Sr}-\mathrm{Pt}^{\mathrm{vii}}$ & $105.89(3)$ \\
\hline $\mathrm{Sr}-\mathrm{Pt}-\mathrm{Sr}^{\mathrm{vi}}$ & $74.11(3)$ & $\mathrm{Pt}^{\mathrm{vi}}-\mathrm{Sr}-\mathrm{Pt}^{\mathrm{vii}}$ & $87.96(4)$ \\
\hline $\mathrm{Cd}^{\mathrm{i}}-\mathrm{Pt}-\mathrm{Sr}^{\mathrm{vii}}$ & $72.78(3)$ & $\mathrm{Pt}^{\mathrm{i}}-\mathrm{Sr}-\mathrm{Cd}^{\mathrm{vii}}$ & $51.57(2)$ \\
\hline $\mathrm{Cd}^{\mathrm{ii}}-\mathrm{Pt}-\mathrm{Sr}^{\mathrm{vii}}$ & $142.88(3)$ & $\mathrm{Pt}^{\mathrm{ii}}-\mathrm{Sr}-\mathrm{Cd}^{\mathrm{vii}}$ & $107.65(4)$ \\
\hline $\mathrm{Cd}-\mathrm{Pt}-\mathrm{Sr}^{\mathrm{vii}}$ & $67.51(3)$ & $\mathrm{Pt}-\mathrm{Sr}-\mathrm{Cd}^{\mathrm{vii}}$ & $138.55(2)$ \\
\hline $\mathrm{Cd}^{\mathrm{iii}}-\mathrm{Pt}-\mathrm{Sr}^{\mathrm{vii}}$ & $135.959(18)$ & $\mathrm{Pt}^{\mathrm{vi}}-\mathrm{Sr}-\mathrm{Cd}^{\mathrm{vii}}$ & $106.76(4)$ \\
\hline $\mathrm{Sr}{ }^{\mathrm{iv}}-\mathrm{Pt}-\mathrm{Sr}^{\mathrm{vii}}$ & $137.64(2)$ & $\mathrm{P}^{\mathrm{vii}}-\mathrm{Sr}-\mathrm{Cd}^{\mathrm{vii}}$ & $51.10(2)$ \\
\hline $\mathrm{Sr}^{\mathrm{v}}-\mathrm{Pt}-\mathrm{Sr}^{\mathrm{vii}}$ & $76.441(19)$ & $\mathrm{Pt}-\mathrm{Sr}-\mathrm{Cd}^{\mathrm{vi}}$ & $107.65(4)$ \\
\hline $\mathrm{Sr}-\mathrm{Pt}-\mathrm{Sr}^{\mathrm{vii}}$ & $74.11(3)$ & $\mathrm{Pt} \mathrm{t}^{\mathrm{ii}}-\mathrm{Sr}-\mathrm{Cd}^{\mathrm{vi}}$ & $51.57(2)$ \\
\hline $\mathrm{Sr}^{\mathrm{vi}}-\mathrm{Pt}-\mathrm{Sr}^{\mathrm{vii}}$ & $87.96(4)$ & $\mathrm{Pt}-\mathrm{Sr}-\mathrm{Cd}^{\mathrm{vi}}$ & $138.55(2)$ \\
\hline $\mathrm{Pt}^{\mathrm{iv}}-\mathrm{Cd}-\mathrm{Pt}^{\mathrm{v}}$ & $103.87(4)$ & $\mathrm{Pt}^{\mathrm{vi}}-\mathrm{Sr}-\mathrm{Cd}^{\mathrm{vi}}$ & $51.10(2)$ \\
\hline $\mathrm{Pt}^{\mathrm{iv}}-\mathrm{Cd}-\mathrm{Pt}$ & $117.50(2)$ & $\mathrm{Pt}^{\mathrm{vii}}-\mathrm{Sr}-\mathrm{Cd}^{\mathrm{vi}}$ & $106.76(4)$ \\
\hline $\mathrm{Pt}^{\mathrm{v}}-\mathrm{Cd}-\mathrm{Pt}$ & $117.50(2)$ & $\mathrm{Cd}^{\mathrm{vii}}-\mathrm{Sr}-\mathrm{Cd}^{\mathrm{vi}}$ & $82.56(4)$ \\
\hline $\mathrm{P}^{\mathrm{tiv}}-\mathrm{Cd}-\mathrm{Pt}^{\mathrm{viii}}$ & $108.97(3)$ & $\mathrm{Pt}^{\mathrm{i}}-\mathrm{Sr}-\mathrm{Cd}^{\mathrm{xii}}$ & $50.65(2)$ \\
\hline $\mathrm{Pt}^{\mathrm{v}}-\mathrm{Cd}-\mathrm{Pt}^{\mathrm{viii}}$ & $108.97(3)$ & $\mathrm{Pt}^{\mathrm{ii}}-\mathrm{Sr}-\mathrm{Cd}^{\mathrm{xii}}$ & $50.65(2)$ \\
\hline $\mathrm{Pt}-\mathrm{Cd}-\mathrm{Pt}^{\mathrm{viii}}$ & $99.68(3)$ & $\mathrm{Pt}-\mathrm{Sr}-\mathrm{Cd}^{\mathrm{xii}}$ & $130.48(4)$ \\
\hline $\mathrm{Pt}^{\mathrm{iv}}-\mathrm{Cd}-\mathrm{Cd}^{\mathrm{ix}}$ & $54.88(2)$ & $\mathrm{Pt}^{\mathrm{vi}}-\mathrm{Sr}-\mathrm{Cd}^{\mathrm{xii}}$ & $109.17(3)$ \\
\hline $\mathrm{Pt}^{\mathrm{v}}-\mathrm{Cd}-\mathrm{Cd}^{\mathrm{ix}}$ & $119.11(5)$ & $\mathrm{P}^{\mathrm{vii}}-\mathrm{Sr}-\mathrm{Cd}^{\mathrm{xii}}$ & $109.17(3)$ \\
\hline $\mathrm{Pt}-\mathrm{Cd}-\mathrm{Cd}^{\mathrm{ix}}$ & $122.75(4)$ & $\mathrm{Cd}^{\mathrm{vii}}-\mathrm{Sr}-\mathrm{Cd}^{\mathrm{xii}}$ & $58.19(3)$ \\
\hline $\mathrm{Pt}^{\mathrm{viii}}-\mathrm{Cd}-\mathrm{Cd}^{\mathrm{ix}}$ & $54.10(3)$ & $\mathrm{Cd}^{\mathrm{vi}}-\mathrm{Sr}-\mathrm{Cd}^{\mathrm{xii}}$ & $58.19(3)$ \\
\hline $\mathrm{Pt}^{\mathrm{iv}}-\mathrm{Cd}-\mathrm{Cd}^{\mathrm{x}}$ & $119.11(5)$ & $\mathrm{Pt}^{\mathrm{i}}-\mathrm{Sr}-\mathrm{Cd}^{\mathrm{i}}$ & $50.41(2)$ \\
\hline $\mathrm{Pt}^{\mathrm{v}}-\mathrm{Cd}-\mathrm{Cd}^{\mathrm{x}}$ & $54.88(2)$ & $\mathrm{P}^{\mathrm{ti}}-\mathrm{Sr}-\mathrm{Cd}^{\mathrm{i}}$ & $105.21(4)$ \\
\hline $\mathrm{Pt}-\mathrm{Cd}-\mathrm{Cd}^{\mathrm{x}}$ & $122.75(4)$ & $\mathrm{Pt}-\mathrm{Sr}-\mathrm{Cd}^{\mathrm{i}}$ & $50.11(2)$ \\
\hline $\mathrm{Pt}^{\mathrm{viii}}-\mathrm{Cd}-\mathrm{Cd}^{\mathrm{x}}$ & $54.10(3)$ & $\mathrm{Pt}^{\mathrm{vi}}-\mathrm{Sr}-\mathrm{Cd}^{\mathrm{i}}$ & $154.30(5)$ \\
\hline $\mathrm{Cd}^{\mathrm{ix}}-\mathrm{Cd}-\mathrm{Cd}^{\mathrm{x}}$ & $84.81(5)$ & $\mathrm{Pt}^{\mathrm{vii}}-\mathrm{Sr}-\mathrm{Cd}^{\mathrm{i}}$ & $90.55(2)$ \\
\hline $\mathrm{Pt}^{\mathrm{iv}}-\mathrm{Cd}-\mathrm{Sr}^{\mathrm{vii}}$ & $167.74(3)$ & $\mathrm{Cd}^{\mathrm{vii}}-\mathrm{Sr}-\mathrm{Cd}^{\mathrm{i}}$ & $92.00(2)$ \\
\hline $\mathrm{Pt}^{\mathrm{v}}-\mathrm{Cd}-\mathrm{Sr}^{\mathrm{vii}}$ & $86.46(2)$ & $\mathrm{Cd}^{\mathrm{vi}}-\mathrm{Sr}-\mathrm{Cd}^{\mathrm{i}}$ & $151.86(5)$ \\
\hline $\mathrm{Pt}-\mathrm{Cd}-\mathrm{Sr}^{\mathrm{vii}}$ & $61.38(3)$ & $\mathrm{Cd}^{\mathrm{xii}}-\mathrm{Sr}-\mathrm{Cd}^{\mathrm{i}}$ & $95.54(3)$ \\
\hline
\end{tabular}




\begin{tabular}{|c|c|c|c|}
\hline $\mathrm{Pt}^{\mathrm{viii}}-\mathrm{Cd}-\mathrm{Sr}^{\mathrm{vii}}$ & $60.64(3)$ & $\mathrm{Pt}-\mathrm{Sr}-\mathrm{Cd}^{\mathrm{ii}}$ & $105.21(4)$ \\
\hline $\mathrm{Cd}^{\mathrm{ix}}-\mathrm{Cd}-\mathrm{Sr}^{\mathrm{vii}}$ & $114.39(5)$ & $\mathrm{Pt} \mathrm{t}^{\mathrm{ii}}-\mathrm{Sr}-\mathrm{Cd}^{\mathrm{ii}}$ & $50.41(2)$ \\
\hline $\mathrm{Cd}^{\mathrm{x}}-\mathrm{Cd}-\mathrm{Sr}^{\mathrm{vii}}$ & $61.52(3)$ & $\mathrm{Pt}-\mathrm{Sr}-\mathrm{Cd}^{\mathrm{ii}}$ & $50.11(2)$ \\
\hline $\mathrm{Pt}^{\mathrm{tiv}}-\mathrm{Cd}-\mathrm{Sr}^{\mathrm{vi}}$ & $86.46(2)$ & $\mathrm{Pt}^{\mathrm{vi}}-\mathrm{Sr}-\mathrm{Cd}^{\mathrm{ii}}$ & $90.55(2)$ \\
\hline $\mathrm{Pt}^{\mathrm{v}}-\mathrm{Cd}-\mathrm{Sr}^{\mathrm{vi}}$ & $167.74(3)$ & $\mathrm{P}^{\mathrm{vii}}-\mathrm{Sr}-\mathrm{Cd}^{\mathrm{ii}}$ & $154.30(5)$ \\
\hline $\mathrm{Pt}-\mathrm{Cd}-\mathrm{Sr}^{\mathrm{vi}}$ & $61.38(3)$ & $\mathrm{Cd}^{\mathrm{vii}}-\mathrm{Sr}-\mathrm{Cd}^{\mathrm{ii}}$ & $151.86(5)$ \\
\hline $\mathrm{Pt}^{\mathrm{viii}}-\mathrm{Cd}-\mathrm{Sr}^{\mathrm{vi}}$ & $60.64(3)$ & $\mathrm{Cd}^{\mathrm{vi}}-\mathrm{Sr}-\mathrm{Cd}^{\mathrm{ii}}$ & $92.00(2)$ \\
\hline $\mathrm{Cd}^{\mathrm{ix}}-\mathrm{Cd}-\mathrm{Sr}^{\mathrm{vi}}$ & $61.52(3)$ & $\mathrm{Cd}^{\mathrm{xii}}-\mathrm{Sr}-\mathrm{Cd}^{\mathrm{ii}}$ & $95.54(3)$ \\
\hline $\mathrm{Cd}^{\mathrm{x}}-\mathrm{Cd}-\mathrm{Sr}^{\mathrm{vi}}$ & $114.39(5)$ & $\mathrm{Cd}-\mathrm{Sr}-\mathrm{Cd}^{\mathrm{ii}}$ & $79.86(4)$ \\
\hline $\mathrm{Sr}^{\mathrm{vii}}-\mathrm{Cd}-\mathrm{Sr}^{\mathrm{vi}}$ & $82.56(4)$ & $\mathrm{Pt}^{\mathrm{i}}-\mathrm{Sr}-\mathrm{Cd}^{\text {viii }}$ & $134.25(2)$ \\
\hline $\mathrm{Pt}^{\mathrm{iv}}-\mathrm{Cd}-\mathrm{Sr}^{\mathrm{xi}}$ & $60.31(2)$ & $\mathrm{Pt}^{\mathrm{tii}}-\mathrm{Sr}-\mathrm{Cd}^{\mathrm{viii}}$ & $134.25(2)$ \\
\hline $\mathrm{Pt}^{\mathrm{v}}-\mathrm{Cd}-\mathrm{Sr}^{\mathrm{xi}}$ & $60.31(2)$ & $\mathrm{Pt}-\mathrm{Sr}-\mathrm{Cd}^{\text {viii }}$ & $88.75(4)$ \\
\hline $\mathrm{Pt}-\mathrm{Cd}-\mathrm{Sr}^{\mathrm{xi}}$ & $175.05(4)$ & $\mathrm{Pt}^{\mathrm{vi}}-\mathrm{Sr}-\mathrm{Cd}^{\mathrm{viii}}$ & $48.75(2)$ \\
\hline $\mathrm{Pt}^{\mathrm{v}}{ }^{\mathrm{vii}}-\mathrm{Cd}-\mathrm{Sr}^{\mathrm{xi}}$ & $85.27(3)$ & $\mathrm{Pt}^{\mathrm{v}}{ }^{\mathrm{vi}}-\mathrm{Sr}-\mathrm{Cd}^{\mathrm{viii}}$ & $48.75(2)$ \\
\hline $\mathrm{Cd}^{\mathrm{ix}}-\mathrm{Cd}-\mathrm{Sr}^{\mathrm{xi}}$ & $60.30(3)$ & $\mathrm{Cd}^{\mathrm{vii}}-\mathrm{Sr}-\mathrm{Cd}^{\text {viii }}$ & 93.99 (3) \\
\hline $\mathrm{Cd}^{\mathrm{x}}-\mathrm{Cd}-\mathrm{Sr}^{\mathrm{xi}}$ & $60.30(3)$ & $\mathrm{Cd}^{\mathrm{vi}}-\mathrm{Sr}-\mathrm{Cd}^{\mathrm{viii}}$ & $93.99(3)$ \\
\hline $\mathrm{Sr}$ vii $-\mathrm{Cd}-\mathrm{Sr}^{\mathrm{xi}}$ & $121.81(3)$ & $\mathrm{Cd}^{\mathrm{xii}}-\mathrm{Sr}-\mathrm{Cd}^{\mathrm{viii}}$ & $140.77(5)$ \\
\hline $\mathrm{Sr}{ }^{\mathrm{vi}}-\mathrm{Cd}-\mathrm{Sr}^{\mathrm{xi}}$ & $121.81(3)$ & $\mathrm{Cd}-\mathrm{Sr}-\mathrm{Cd}^{\mathrm{v}}{ }^{\mathrm{viii}}$ & $113.98(3)$ \\
\hline $\mathrm{Pt}^{\mathrm{iv}}-\mathrm{Cd}-\mathrm{Sr}^{\mathrm{v}}$ & $120.35(4)$ & $\mathrm{Cd}^{\mathrm{ii}}-\mathrm{Sr}-\mathrm{Cd}^{\text {viii }}$ & $113.98(3)$ \\
\hline $\mathrm{Pt}^{\mathrm{v}}-\mathrm{Cd}-\mathrm{Sr}^{\mathrm{v}}$ & $59.65(2)$ & $\mathrm{Pt}^{\mathrm{i}}-\mathrm{Sr}-\mathrm{Sr}^{\mathrm{vi}}$ & $152.61(6)$ \\
\hline $\mathrm{Pt}-\mathrm{Cd}-\mathrm{Sr}^{\mathrm{v}}$ & $59.46(3)$ & $\mathrm{Pt}^{\mathrm{ii}}-\mathrm{Sr}-\mathrm{Sr}^{\mathrm{vi}}$ & $94.42(2)$ \\
\hline $\mathrm{Pt}^{\mathrm{viii}}-\mathrm{Cd}-\mathrm{Sr}^{\mathrm{v}}$ & $130.66(3)$ & $\mathrm{Pt}-\mathrm{Sr}-\mathrm{Sr}^{\mathrm{vi}}$ & $53.25(3)$ \\
\hline $\mathrm{Cd}^{\mathrm{ix}}-\mathrm{Cd}-\mathrm{Sr}^{\mathrm{v}}$ & $175.11(5)$ & $\mathrm{Pt}^{\mathrm{vi}}-\mathrm{Sr}-\mathrm{Sr}^{\mathrm{vi}}$ & $52.64(2)$ \\
\hline $\mathrm{Cd}^{\mathrm{x}}-\mathrm{Cd}-\mathrm{Sr}^{\mathrm{v}}$ & $97.51(2)$ & $\mathrm{Pt}^{\mathrm{vii}}-\mathrm{Sr}-\mathrm{Sr}^{\mathrm{vi}}$ & $101.34(5)$ \\
\hline $\mathrm{Sr}^{\mathrm{vii}}-\mathrm{Cd}-\mathrm{Sr}^{\mathrm{v}}$ & $70.47(2)$ & $\mathrm{Cd}^{\mathrm{vii}}-\mathrm{Sr}-\mathrm{Sr}^{\mathrm{vi}}$ & $149.28(6)$ \\
\hline $\mathrm{Sr}{ }^{\mathrm{vi}}-\mathrm{Cd}-\mathrm{Sr}^{\mathrm{v}}$ & $120.84(3)$ & $\mathrm{Cd}^{\mathrm{vi}}-\mathrm{Sr}-\mathrm{Sr}^{\mathrm{vi}}$ & $95.53(3)$ \\
\hline $\mathrm{Sr}^{\mathrm{xi}}-\mathrm{Cd}-\mathrm{Sr}^{\mathrm{v}}$ & $117.17(3)$ & $\mathrm{Cd}^{\mathrm{xii}}-\mathrm{Sr}-\mathrm{Sr}^{\mathrm{vi}}$ & $144.11(3)$ \\
\hline $\mathrm{Pt}^{\mathrm{iv}}-\mathrm{Cd}-\mathrm{Sr}^{\mathrm{iv}}$ & $59.65(2)$ & $\mathrm{Cd}-\mathrm{Sr}-\mathrm{Sr}^{\mathrm{ii}}$ & $102.75(5)$ \\
\hline $\mathrm{Pt}^{\mathrm{v}}-\mathrm{Cd}-\mathrm{Sr}^{\mathrm{iv}}$ & $120.35(4)$ & $\mathrm{Cd}^{\mathrm{ii}}-\mathrm{Sr}-\mathrm{Sr}^{\mathrm{vi}}$ & $58.53(3)$ \\
\hline $\mathrm{Pt}-\mathrm{Cd}-\mathrm{Sr}^{\mathrm{iv}}$ & $59.46(3)$ & $\mathrm{Cd}^{\mathrm{viii}}-\mathrm{Sr}-\mathrm{Sr}^{\mathrm{vi}}$ & $55.44(3)$ \\
\hline
\end{tabular}

Symmetry codes: (i) $-x+1 / 2,-y+1, z+1 / 2$; (ii) $-x+1 / 2,-y, z+1 / 2$; (iii) $x+1 / 2, y,-z+1 / 2$; (iv) $-x+1 / 2,-y, z-1 / 2$; (v) $-x+1 / 2,-y+1, z-1 / 2$; (vi) $-x,-y,-z+1$; (vii) $-x,-y+1,-z+1$; (viii) $x-1 / 2, y,-z+1 / 2$; (ix) $-x,-y,-z$; (x) $-x,-y+1,-z$; (xi) $x, y, z-1$; (xii) $x, y, z+1$. 